\title{
MODELOS DE SIMULACIÓN DEL CRECIMIENTO DE LECHUGA EN RESPUESTA A LA FERTILIZACIÓN ORGÁNICA Y MINERAL
}

\section{SIMULATION MODELS OF LETTUCE GROWTH IN RESPONSE TO ORGANIC AND MINERAL FERTILIZATION}

\author{
Victorino D. Jiménez-Morales ${ }^{1}$, Libia I. Trejo-Téllez ${ }^{1 *}$, Fernando C. Gómez-Merino² \\ y Víctor H. Volke-Haller ${ }^{1}$
}

${ }^{1}$ Campus Montecillo, Colegio de Postgraduados. km 36.5 Carr. México-Texcoco. 56230, Montecillo, Edo. de México. ${ }^{2}$ Campus Córdoba, Colegio de Postgraduados. km 348 Carr. Córdoba-Veracruz. 94490, Amatlán de los Reyes, Veracruz.

${ }^{*}$ Autora responsable (tlibia@colpos.mx)

\section{RESUMEN}

Se evaluó el efecto de la aplicación simple y combinada de extracto de vermicompost (fertilización orgánica) y solución de Steiner (fertilización mineral), en la producción de biomasa, área foliar, número de hojas y altura de planta de lechuga (Lactuca sativa var. longifolia) cv. Parris Island en hidroponía. Los resultados mostraron que la aplicación individual de dosis bajas de extracto de vermicompost incrementaron las variables medidas. Sin embargo, este efecto se tornó negativo conforme se incrementó la concentración del extracto de vermicompost. También se observaron efectos positivos al aplicar bajas concentraciones de extracto de vermicompost adicionadas con bajas concentraciones de la solución de Steiner. No obstante, al aumentar ambos factores se observaron efectos negativos, los cuales fueron más evidentes conforme se incrementó la concentración del extracto de vermicompost. Los efectos negativos están asociados a altos valores de conductividad eléctrica en los tratamientos con los niveles más altos de extracto de vermicompost y de la solución Steiner.

Palabras clave: Lactuca sativa, extracto de vermicompost, solución de Steiner, modelaje matemático.

\section{SUMMARY}

The effect of the simple or combined application of vermicompost tea (organic fertilization) and Steiner's solution (mineral fertilization) at five concentrations $(0,25,50,75$ and $100 \%)$, on biomass production, leaf area, leaf number and plant height of lettuce (Lactuca sativa var. longifolia) cv. 'Parris Island' was evaluated under hydroponics. Results showed that the simple application of vermicompost tea at low concentration improved all variables measured. However, this effect turned negative as the concentration of vermicompost tea increased in the nutrient solution. Also, it was observed that low concentrations of vermicompost tea in combination with low levels of Steiner's solution improved plant growth. Nevertheless, as concentration of both factors increased in the hydroponic culture, negative effects on all variables measured were detected, and such effects were more evident when vermicompost tea concentrations were high. The negative effects may be associated to high electric conductivity observed in treatments with the highest levels of both the vermicompost tea and the nutrient solution used.

Index words: Lactuca sativa, vermicompost tea, Steiner solution, mathematical modelling.

\section{INTRODUCCIÓN}

La investigación en vermicompost se ha enfocado principalmente al estudio de los efectos de la fracción sólida sobre germinación, crecimiento y control de enfermedades de las plantas, mientras que en los últimos años se ha comenzado a estudiar la fracción líquida de este material (Edwards et al., 2006). Es posible que el extracto de vermicompost presente características químicas y microbiológicas similares a la fracción sólida, debido a la probable incorporación de nutrimentos solubles, microorganismos benéficos, ácidos húmicos y fúlvicos, fitohormonas y reguladores de crecimiento vegetal durante el proceso de elaboración de los extractos (Schmitz, 2002). Así, González-Solano et al. (2013) establecieron relaciones entre características químicas de los extractos o tés y el origen del vermicompost usado en la extracción, la relación agua:vermicompost y el tiempo de incubación.

El uso de la fracción líquida de vermicompost en México es escaso y no existe suficiente soporte técnico que apoye su empleo en la producción agrícola. La importancia de estudiar su posible uso radica en que estos extractos ofrecen una alternativa viable para reducir las dosis de fertilizantes y otros insumos agrícolas, dado que contienen nutrimentos esenciales, microorganismos benéficos, sustancias promotoras del crecimiento, y ácidos fúlvicos y húmicos (Pant et al., 2009; Albert et al., 2012), lo cual permitiría disminuir costos de producción y reducir la emisión de contaminantes al ambiente.

Por tanto, este estudio tuvo la finalidad de evaluar el uso del extracto de vermicompost combinado en diferentes proporciones con solución nutritiva en condiciones de hidroponía bajo invernadero, en la producción de lechuga (Lactuca sativa var. longifolia) cultivar Parris Island y definir modelos de simulación del crecimiento de este cultivo. 


\section{MATERIALES Y MÉTODOS}

El experimento se condujo en un invernadero cubierto con plástico calibre 600, ubicado en Montecillo, Texcoco, Estado de México, con plántulas de lechuga cv. Parris Island, obtenidas de siembra de semillas en charolas de germinación usando turba como sustrato.

El diseño experimental usado fue completamente al azar factorizado incompleto $\left(5^{2}\right)$. Los factores evaluados fueron: extracto de vermicompost (V) y solución nutritiva de Steiner (S) (Steiner, 1984), ambos en cinco niveles (0, 25, 50, 75 y $100 \%$, v/v). Al aplicar el cuadrado doble, se evaluaron solo 13 tratamientos en lugar de los 25 resultantes del factorial completo para economizar espacio y gastos; los 13 tratamientos presentaron una misma área de exploración. Los tratamientos evaluados fueron: $\mathrm{V}_{0} \mathrm{~S}_{0}, \mathrm{~V}_{0} \mathrm{~S}_{50}, \mathrm{~V}_{0} \mathrm{~S}_{100}, \mathrm{~V}_{25} \mathrm{~S}_{25}$, $\mathrm{V}_{25} \mathrm{~S}_{75}, \mathrm{~V}_{50} \mathrm{~S}_{0}, \mathrm{~V}_{50} \mathrm{~S}_{50}, \mathrm{~V}_{50} \mathrm{~S}_{100}, \mathrm{~V}_{75} \mathrm{~S}_{25}, \mathrm{~V}_{75} \mathrm{~S}_{75}, \mathrm{~V}_{100} \mathrm{~S}_{0}, \mathrm{~V}_{100} \mathrm{~S}_{50} \mathrm{y}$ $\mathrm{V}_{100} \mathrm{~S}_{100}$, cada uno con tres repeticiones, y cada repetición con seis plantas.

El almácigo se estableció en charolas de plástico de 200 cavidades y se regó diario con agua destilada. Los riegos se aplicaron con la solución de Hoagland a 25 \% (Hoagland y Arnon, 1950), una vez que las plántulas presentaron las primeras dos hojas verdaderas. El trasplante se realizó 27 $\mathrm{d}$ después de la siembra, en bolsas de plástico negro $(25 \mathrm{x}$ $25 \mathrm{~cm}$ ) rellenadas con tezontle (8 $\mathrm{mm}$ de diámetro) como sustrato.

El extracto de vermicompost se obtuvo solubilizando vermicompost maduro $(\mathrm{C} / \mathrm{N}=12.5)$ con una solución de $\mathrm{KOH} 1 \%$ usando una relación de 1:10 (KOH $1 \%$ :vermicompost). Se dejó reposar la suspensión durante $15 \mathrm{~d}$, para posteriormente eliminar las partículas sólidas por decantación y filtración. Las concentraciones de nutrimentos en el extracto de vermicompost fueron en $\mathrm{mg} \mathrm{L}^{-1}$ : N 260, P 147.9, $\mathrm{K}$ 4397.2, Ca 88.96, Mg 13.4, Fe 9.9, Cu 2.0, Zn 0.68, Mn 0.64 , B 1.0; mientras que la de $\mathrm{Na} 57.8$.

Las soluciones para los diferentes tratamientos se complementaron con micronutrimentos en las siguientes concentraciones (mg L-1): Fe 4.98, Mn 2.33, Zn 0.47, Cu 0.19, B 0.43 y Mo 0.17; el pH de éstas se ajustó a 5.8 y se les determinó la conductividad eléctrica (CE). Las CE fueron: 0, $3.78,6.84,1.95,5.77,0.67,3.88,6.97,2.63,5.68,1.28,4.10 \mathrm{y}$ $7.10 \mathrm{dS} \mathrm{m} \mathrm{m}^{-1}$ para los tratamientos $\mathrm{V}_{0} \mathrm{~S}_{0}, \mathrm{~V}_{50} \mathrm{~S}_{0}, \mathrm{~V}_{100} \mathrm{~S}_{0}, \mathrm{~V}_{25} \mathrm{~S}_{25}$, $\mathrm{V}_{75} \mathrm{~S}_{25}, \mathrm{~V}_{0} \mathrm{~S}_{50}, \mathrm{~V}_{50} \mathrm{~S}_{50}, \mathrm{~V}_{100} \mathrm{~S}_{50}, \mathrm{~V}_{25} \mathrm{~S}_{75}, \mathrm{~V}_{75} \mathrm{~S}_{75}, \mathrm{~V}_{0} \mathrm{~S}_{10}, \mathrm{~V}_{50} \mathrm{~S}_{100}$, $\mathrm{V}_{100} \mathrm{~S}_{100}$, respectivamente.

A los 53 d después del trasplante se midieron las siguientes variables: altura de planta (AP) desde el punto de inserción de los cotiledones hasta la punta de la hoja más larga; número de hojas $(\mathrm{NH})$ con excepción de las de la envoltura floral; área foliar total (AF) con un integrador de área foliar LICOR ${ }^{\circledR}$ modelo LI-3000 (Lincoln, NE, Estados Unidos) sin incluir las hojas de la envoltura floral. Después, la parte aérea de la lechuga (vástago) fue secada a $60^{\circ} \mathrm{C}$ en una estufa marca CLIMATEST ${ }^{\circledR}$ modelo Blue M SW-17TA (San Francisco, CA, Estados Unidos), durante $72 \mathrm{~h}$ y luego se pesó en una balanza digital OHAUS ${ }^{\circledR}$ modelo GT410D (Parsippany, NJ, Estados Unidos) para obtener el peso seco de parte aérea (PSPA).

Las variables evaluadas fueron sometidas a un análisis de regresión mediante el procedimiento de eliminación regresiva (PROC Backward de SAS; SAS Institute), para estimar modelos matemáticos de la función de producción (relación entre factores de estudio V y S, y la variable respuesta), a partir de un modelo inicial completo que incluye todas las variables independientes $\left(Y=\beta_{0}+\beta_{1} S+\beta_{2} V+\beta_{3} S V+\beta_{4} S^{2}\right.$ $\left.+\beta_{5} V^{2}+\beta_{6} S^{2} V+\beta_{7} S V^{2}+\beta_{8} S^{2} V^{2}+e\right)$, con base en las gráficas de respuesta, según el diseño de tratamientos del cuadrado doble. El análisis se realizó con el paquete estadístico SAS (SAS Institute, 2003). De acuerdo con el procedimiento Backward, una significancia de 0.10 se considera criterio de permanencia de las variables (nivel de probabilidad que maneja el programa SAS de forma automática); sin embargo, el modelo matemático final seleccionado fue el de menor cuadrado medio del error (CME).

\section{RESULTADOS Y DISCUSIÓN}

Las funciones matemáticas de respuesta estimadas consideraron los efectos tanto simples (efectos de la aplicación individual del extracto de vermicompost y de la solución nutritiva universal de Steiner), y los de interacción (efecto combinado de ambos materiales) que resultaron significativos $(\mathrm{P}<0.05)$ de los factores experimentales. Para este trabajo de investigación, los modelos de regresión estimados resultaron significativos al nivel de probabilidad de 0.05 ; sin embargo, varios de ellos presentaron valores relativamente bajos de $\mathrm{R}^{2}$. En dichos modelos $V$ representó el factor extracto de vermicompost y $\mathrm{S}$ el factor solución de Steiner.

Peso seco de parte aérea (PSPA). A continuación se representa el modelo de función de respuesta del peso de biomasa de hojas de lechuga:

$P S P A=5.499+0.105 S+0.0483 \mathrm{~V}-0.00000708 S^{2}$

$\left(\mathrm{CME}=4.073 ; \mathrm{P}<0.0001 ; \mathrm{R}^{2}=0.73\right)$

El peso de biomasa mostró una repuesta positiva a la aplicación de solución de Steiner en ausencia de extracto de vermicompost, ya que el valor de esta variable aumentó conforme se incrementaban los niveles de solución nutritiva (Figura 1A). También hubo efecto positivo por la aplicación de extracto de vermicompost en ausencia de solución 
de Steiner, porque promovió el aumento de peso de biomasa (Figura 1B). Por otro lado, el único término de interacción significativo entre la solución de Steiner y el extracto de vermicompost, fue a nivel cuadrático, y tuvo un efecto negativo, por lo que la aplicación conjunta de ambos factores no incrementó el peso de biomasa, efecto que resultó más notorio a los niveles más altos del factor extracto de vermicompost.

Área foliar (AF). El modelo de función de respuesta para el área foliar fue:

$A F=698.539+23.908 S+11.12 V-0.00176 S V^{2}$

$\left(\mathrm{CME}=208938 ; \mathrm{P}<0.0001 ; \mathrm{R}^{2}=0.72\right)$

La aplicación de solución de Steiner en ausencia de ex- tracto de vermicompost tuvo efecto positivo en el área foliar (Figura 2A). Así mismo, el extracto de vermicompost, en ausencia de solución nutritiva tuvo un efecto similar al de la solución de Steiner en la cantidad de área foliar (Figura 2B). Por otra parte, se observó un efecto de interacción negativo entre las variables de estudio; esto es, el valor del área foliar no mostró incrementos adicionales a niveles altos de la aplicación combinada de los dos factores experimentales, teniendo más influencia sobre esta variable el factor extracto de vermicompost.

Número de hojas (NH). La función de respuesta para el número de hojas por planta fue:

$N H=19.919+0.238 S+0.198 \mathrm{~V}-0.000963 S^{2}-0.00123 \mathrm{~V}^{2}-0.000998 S \mathrm{~V}$ $\left(\mathrm{CME}=7.328 ; \mathrm{P}<0.0001 ; \mathrm{R}^{2}=0.74\right)$
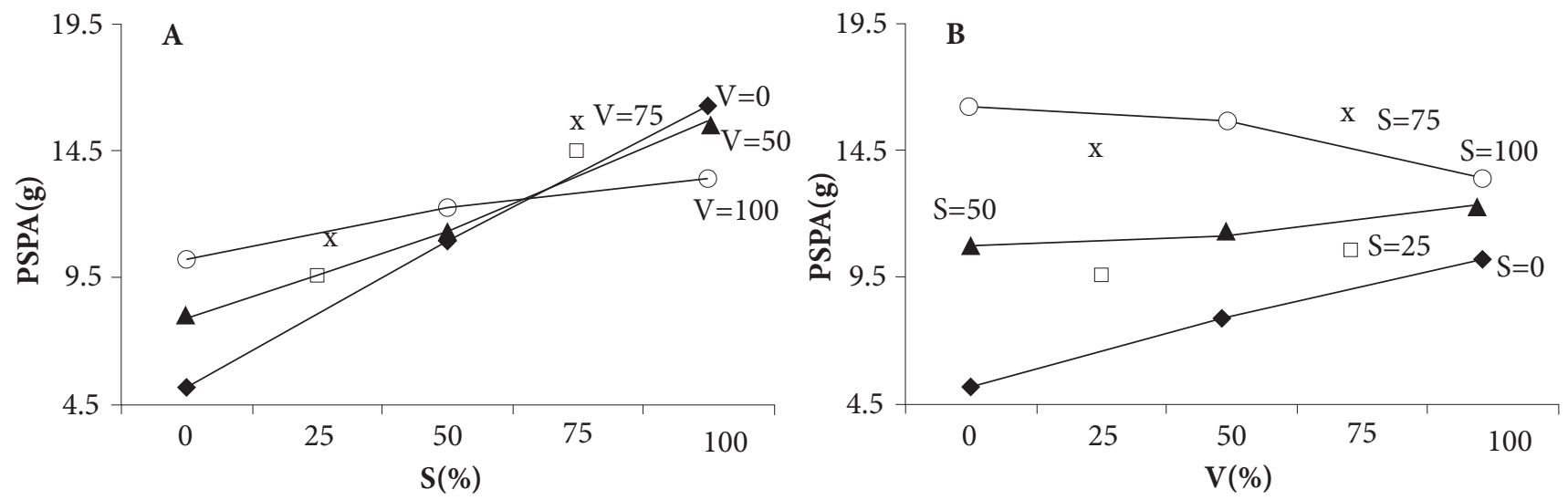

Figura 1. Respuesta del peso seco de parte aérea (PSPA). A) A la aplicación de solución de Steiner (S) a niveles constantes de vermicompost (V). B) A la aplicación de extracto de vermicompost (V) a niveles constantes de solución de Steiner (S). PSPA $=5.499+0.105 S+0.0483 V-0.00000708 S V^{2}\left(\mathrm{CME}=4.073 ; \mathrm{P}<0.0001 ; \mathrm{R}^{2}=0.73\right)$.
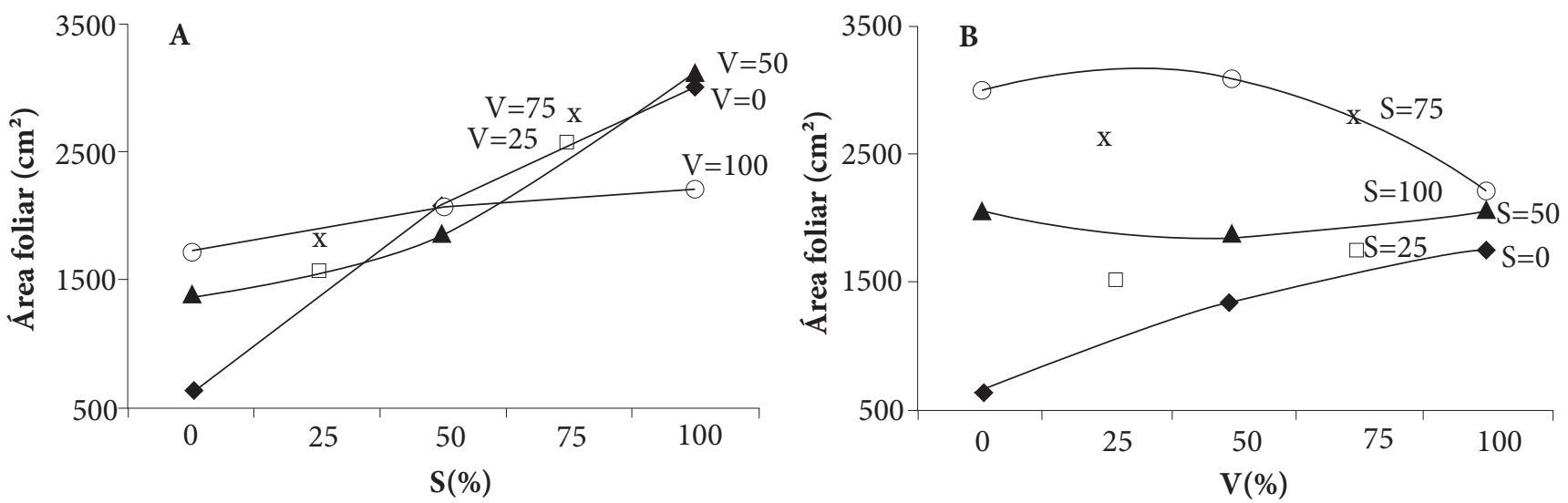

Figura 2. Respuesta del área foliar (AF). A) A la aplicación de solución de Steiner (S) a niveles constantes de vermicompost $(V)$. B) A la aplicación de extracto de vermicompost $(\mathrm{V})$ a niveles constantes de solución de Steiner $(\mathrm{S}) . A F=698.539+23.908$ $S+11.12 V-0.00176 S V^{2} .\left(\mathrm{CME}=208938 ; \mathrm{P}<0.0001 ; \mathrm{R}^{2}=0.72\right)$. 
El número de hojas se incrementó significativamente con la aplicación de niveles bajos de solución de Steiner en ausencia de extracto de vermicompost, pero para los niveles más altos de este mismo factor ya no se observaron incrementos significativos de la cantidad de hojas (Figura 3A). El extracto de vermicompost en ausencia de solución de Steiner también tuvo un efecto significativo positivo a niveles bajos, mientras que a los niveles más altos el efecto fue negativo, al igual que se observó con el factor solución de Steiner (Figura 3B). La aplicación conjunta de ambos factores también ocasionó un efecto negativo, de manera que los incrementos en número de hojas logrados con los factores principales dejaron de ser significativos.

Altura de planta (AP). La función de respuesta de altura de planta quedó representada por:

$A P=15.018+0.1405 S-0.000574 S^{2}+0.000377 V^{2}-0.000415 S V$

$\left(\mathrm{CME}=1.958 ; \mathrm{P}<0.0001 ; \mathrm{R}^{2}=0.79\right)$

Las concentraciones bajas de la solución de Steiner aplicadas en forma individual afectaron positivamente a esta variable, mientras que con niveles altos el efecto se tornó negativo (Figura 4A). Asimismo, la altura fue afectada significativamente con la sola aplicación de extracto de vermicompost, ya que aumentó su valor conforme se incrementaba la dosis de este factor experimental (Figura 4B). Se presentó una interacción negativa entre la solución de Steiner y el extracto de vermicompost de modo que la altura no tuvo incrementos significativos con la aplicación conjunta de ambos factores.

Las diferentes variables evaluadas tuvieron un comportamiento similar desde el punto de vista biológico, ya que se encontraron efectos positivos con la aplicación de cada factor por separado. Para la solución de Steiner ello se explica porque contiene los elementos esenciales para la nutrición de las plantas. Sin embargo, el extracto de vermicompost cuyo aporte nutrimental no es alto (Moreno y Aguilera, 2003), promovió el crecimiento de lechuga, sobre todo cuando fue suministrado a niveles bajos.

En cambio, el efecto de interacción entre ambos factores fue negativo, ya que las plantas no mostraron incrementos adicionales conforme se elevaba el nivel de la aplicación de cualquiera de los dos factores, efecto que fue más notorio a los niveles más altos del factor extracto de vermicompost. Resultados similares se han reportado para la aplicación de vermicompost (fracción sólida) al suelo o como sustrato en tomate (Solanum lycopersicum L.), chile (Capiscum annuum L.), fresa (Fragaria $x$ ananassa Duch.) y clavel (Dianthus caryophyllus L.) (Atiyeh et al., 2002; Arancon et al., 2004), estudios en los que se lograron incrementos en germinación y en variables de crecimiento (peso de biomasa, altura de planta, área foliar, número de hojas y número de botones florales) con niveles bajos de aplicación, pero no con niveles altos de aplicación de vermicompost, comportamiento similar con los resultados aquí obtenidos.

En dichos experimentos previos la aplicación de vermicompost fue independiente de los requerimientos nutricionales, porque todas las plantas fueron regadas con una solución nutritiva que contuviera dichos elementos, para asegurar que las diferencias en crecimiento no fueran por causas nutrimentales. Atiyeh et al. (2002), al evaluar la aplicación de ácidos húmicos extraídos de vermicompost en tomate y pepino (Cucumis sativus L.), encontraron respuestas similares a las aquí reportadas. Por su parte, Chen y Aviad (1990) y Turkmen et al. (2004) reportaron que las curvas de crecimiento de plantas tratadas con sustancias húmicas
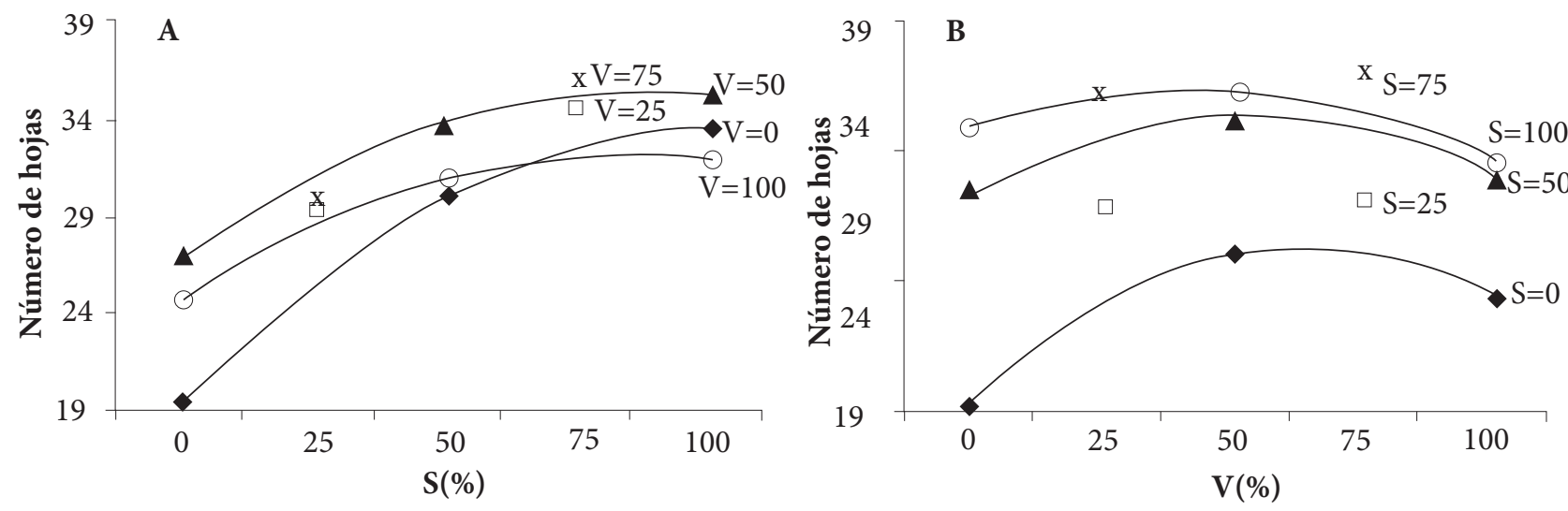

Figura 3. Respuesta del número de hojas (NH). A) A la aplicación de solución de Steiner (S) a niveles constantes de vermicompost $(\mathrm{V})$. B) A la aplicación de extracto de vermicompost $(\mathrm{V})$ a niveles constantes de solución de Steiner (S). $N H=19.919$ $+0.238 S+0.198 V-0.000963 S^{2}-0.00123 V^{2}-0.000998 S V\left(\mathrm{CME}=7.328 ; \mathrm{P}<0.0001 ; \mathrm{R}^{2}=0.74\right)$. 

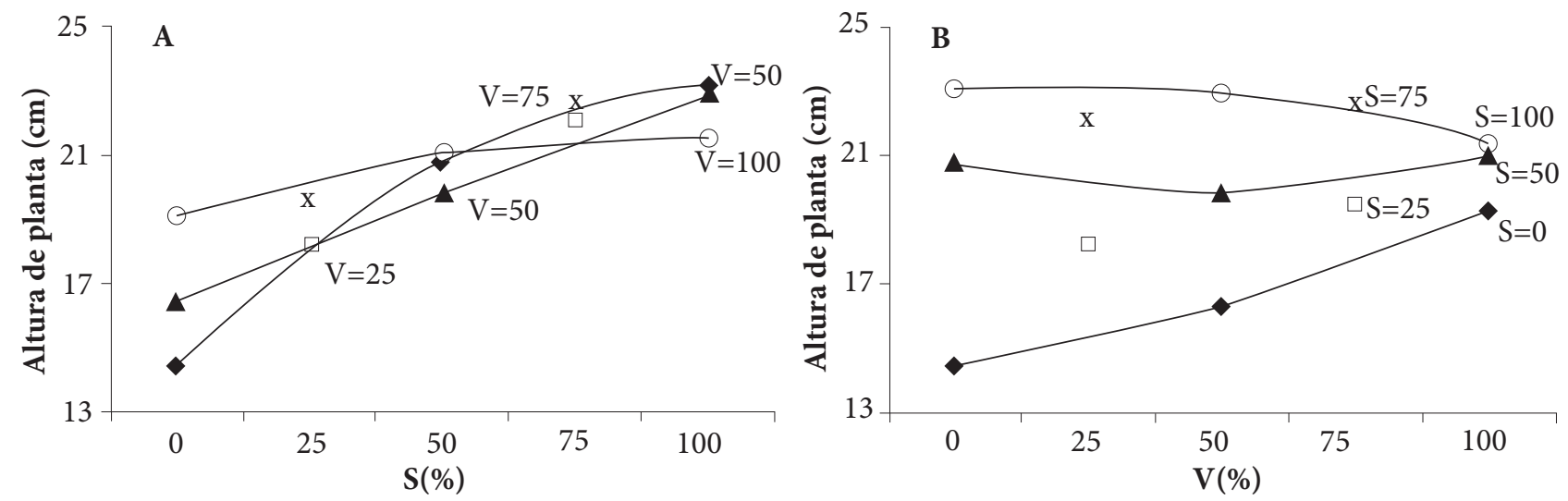

Figura 4. Respuesta de la altura de planta (AP). A) A la aplicación de solución de Steiner (S) a niveles constantes de vermicompost (V). B) A la aplicación de extracto de vermicompost (V) a niveles constantes de solución de $\mathrm{Steiner}(\mathrm{S}) . A P=15.018$ $+0.1405 S-0.000574 S^{2}+0.000377 V^{2}-0.000415 S V .\left(\mathrm{CME}=1.958 ; \mathrm{P}<0.0001 ; \mathrm{R}^{2}=0.79\right)$.

mostraron incrementos progresivos conforme se incrementaba la concentración de estas sustancias, pero que a los niveles más altos de aplicación la respuesta decreció.

El vermicompost contiene una gran cantidad de sustancias húmicas, en el intervalo entre 20 y 40 \% (Capistrán et al., 2001), por lo que la respuesta a la aplicación de extracto de vermicompost pudiera ser atribuida a las sustancias húmicas u otras asociadas con los ácidos húmicos que contienen. Por otro lado, los resultados de los citados estudios y los que aquí se reportan sugieren que el extracto de vermicompost presenta efectos similares que el vermicompost mismo (fracción sólida). Lo anterior coincide con los hallazgos de Edwards et al. (2006), quienes reportan que el extracto de vermicompost presenta características químicas y microbiológicas similares a la fracción sólida de vermicompost, debido a la probable incorporación de nutrimentos solubles, microorganismos benéficos, ácidos húmicos y fúlvicos y de reguladores del crecimiento vegetal durante el proceso de elaboración de los extractos.

La hipótesis más aceptada de la influencia positiva de las sustancias húmicas a bajas concentraciones (Atiyeh et al., 2002) se refiere por un lado a un efecto directo de naturaleza hormonal que puede simular modos de acción de reguladores de crecimiento vegetal (Varanini y Pinton, 2001; Atiyeh et al., 2002; Nardi et al., 2002; Arancon et al., 2004), a los reguladores de crecimiento vegetal adsorbidos en los humatos (Capistrán et al., 2001; Gajalakshmi et al., 2001; Atiyeh et al., 2002; Quaggiotti et al., 2004), o a los reguladores producidos por microorganismos asociados a las lombrices composteras (Capistrán et al., 2001; Arancon et al., 2004).
Del mismo modo, el decremento del crecimiento a niveles altos de vermicompost es atribuido a la alta concentración de sales, pobre aereación y porosidad del medio, toxicidad inducida por metales pesados o a la presencia de sustancias fitotóxicas (Atiyeh et al., 2002; Arancon et al., 2004).

La hipótesis del efecto directo de naturaleza hormonal pareciera estar más acorde con los resultados de la presente investigación, ya que el crecimiento de la lechuga se llevó a cabo en un sustrato sin problemas de porosidad ni de aireación (se usó granulometría < de $8 \mathrm{~mm}$ ).

En este experimento, los tratamientos con los niveles más altos de vermicompost $\left(\mathrm{V}_{100} \mathrm{~S}_{50} \mathrm{y} \mathrm{S}_{100} \mathrm{~V}_{100}\right)$ presentaron conductividades de 6.9 y $7.1 \mathrm{dS} \mathrm{m}^{-1}$ respectivamente, y en el extracto de vermicompost sin diluir de $11 \mathrm{dS} \mathrm{m}^{-1}$, por lo que no se descarta que las altas C.E. hayan ocasionado los efectos negativos en altas dosis de extracto de vermicompost.

Se concluye que el extracto de vermicompost aplicado en dosis baja y media ( 25 y $50 \%$, respectivamente), incrementa el crecimiento de lechuga medido como área foliar, número de hojas y biomasa. La ganancia en altura se acentúa al aplicar niveles altos del extracto.

La solución de Steiner también incrementa la producción de biomasa, área foliar, altura y número de hojas de la lechuga crecida en medio hidropónico.

En cambio, la aplicación combinada de extracto de vermicompost y solución de Steiner en la producción hidropónica de lechuga reduce los valores de peso de biomasa, área foliar, altura de planta y número de hojas conforme se incrementa el nivel de la dosis del extracto de vermicompost. 


\section{BIBLIOGRAFÍA}

Albert N., K. Nazaire, K. Hartmut (2012) The relative effects of compost and non-aerated compost tea in reducing disease symptoms and improving tuberization of Solanum tuberosum in the field. International Journal of Agriculture: Research and Review 2:504-512.

Arancon N. Q., C. A. Edwards, R. Atiyeh, J. D. Metzger (2004) Effects of vermicomposts produced from food waste on the growth and yield of greenhouse peppers. Bioresource Technology 93:139144.

Atiyeh R. M., S. Lee, C. A. Edwards, N. Q. Arancon, J. D. Metzger (2002) The influence of humic acids derived from earthworm-processed organic wastes on plant growth. Bioresource Technology 84:7-14.

Capistrán F., E. Aranda, J. C. Romero (2001) Manual de Reciclaje, Compostaje, y Lombricompostaje. Instituto de Ecología, A. C. Xalapa, Veracruz, México. 150 p.

Chen Y., T. Aviad (1990) Effects of humic substances on plant growth. In: Humic Substances in Soil and Crop Sciences. P. McCarthy, C. E. Clapp, R. L. Malcom and P. R. Bloom (eds). American Society of Agronomy. Madison, WI, USA. pp:161-186.

Edwards C. A., Q. N. Arancon, S. Greytak (2006) Effects of vermicompost teas on plant growth and disease. BioCycle 47:28-31.

Gajalakshmi S., E. V. Ramasamy, S. A. Abbasi (2001) Potential of two epigeic and two anecic earthworm species in vermicomposting of water hyacinth. Bioresource Technology 76:177-181.

González-Solano K. D., M. N. Rodríguez-Mendoza, L. I. Trejo-Téllez, J. Sánchez-Escudero, J. L. García-Cué (2013) Propiedades químicas de tés de vermicompost. Revista Mexicana de Ciencias Agrícolas 5:901-911.

Hoagland D. R., D. I. Arnon (1950) The water-culture method for growing plant without soil. College of Agriculture. University of California. Berkeley, CA, USA. Circular 347.
Moreno R. A., G. S. Aguilera (2003) Efecto de la vermicomposta en chile chilaca (Capsicum annuum L.) bajo condiciones de invernadero. Departamento de Suelos, Unidad Laguna, Universidad Autónoma Agraria Antonio Narro. Torreón, Coahuila, México. 5 p.

Nardi S., D. Pizzeghello, A. Muscolo, A. Vianello (2002) Physiological effects of humic substances on higher plants. Soil Biology and Biochemistry 34:1527-1536.

Pant A. P., K. T. J. Radovich, V. Hue, T. S. Talcott, A. K. Krenek (2009) Effects of Vermicompost tea (Aqueous extract) on Pak Choi Yield, Quality, and on Soil Biological properties. Compost Science and Utilization 19:279-292.

Quaggiotti S., B. Ruperti, D. Pizzeghello, O. Francioso, V. Tugnoli, S. Nardi (2004) Effect of low molecular size humic substances on nitrate uptake and expression of genes involved in nitrate transport in maize (Zea mays L.). Journal of Experimental Botany 55:803-813.

SAS Institute (2003) SAS Institute ver. 9.1.3. Cary, N. C., USA.

Schmitz J. (2002) Compost teas work on center pivot farm. Capitol Press. Albany, NY, USA. 20 p.

Steiner A. (1984) The universal nutrient solution. In: ISOSC Proceedings 6th International Congress on Soilless Culture. The Nether lands. pp:633-649.

Turkmen Ö., A. Dursun, M. Turan, C. Erdine (2004) Calcium and humic acids affect seed germination, growth, and nutrient content of tomato (Lycopersicon esculentum L.) seedlings under saline soil conditions. Acta Agriculturae Scandinavica, Section B-Soil and Plant 54:168-174.

Varanini Z., R. Pinton (2001) Direct versus indirect effects of soil humic substances on plant growth and nutrition. In: The rhizosphere. Biochemistry and organic substances at the soil-plant interface. R Pinton, Z Varanini, P Nannipieri (eds.). Books in Soil, Plants and Environment. Marcel Dekker, Inc. New York, NY, USA. pp:141-157. 